\title{
Early evolution of resistance to oxidative stress after sub- arachnoid hemorrhages: A cohort study of 50 patients
}

\author{
Patrice Thierry $^{*}$, Bertrand Rozec ${ }^{1}$, Romain Bourcier ${ }^{2}$, Hubert Desal $^{2}$ and Yvonnick Blanloeil ${ }^{1}$ \\ ${ }^{1}$ Anesthesiology and Intensive Care, Laënnec Hospital, Nantes, France \\ ${ }^{2}$ Neuroradiology, Laënnec Hospital, Nantes, France
}

\begin{abstract}
Abbreviations: AUC: Area Under the Curve, C-RP: C Reactive Protein, DCF: Dichlorofluorescein (oxidized, fluorescent), DCF-DA: Dichlorofluorescein Diacetate, DCFH: Dichlorofluorescein (reduced, non fluorescent), ICA: Intracranial Aneurism, NLR: Neutrophil-to lymphocyte ratio, NMR: Nuclear Magnetic Resonance, O.D: Optical density, P S100 B: Protein S100 beta, RB: Rose Bengal, ROS: Reactive Oxygen Species, RROS: Resistance to ROS , ${ }^{1} \mathrm{O}_{2:}$ Singlet oxygen, $\mathrm{SAH}$ : Subarachnoid hemorrhages, WFNS: World Federation of Neurologic Surgeons.
\end{abstract}

Background and purpose: The role of Reactive Oxygen Species (ROS) is suspected during subarachnoid haemorrhage. We analysed the early changes in the resistance to ROS (RROS) as a function of the vital prognosis at Day 15 together with other prognostic markers.

Methods: Fifty patients had been successively recruited and RROS has been measured using a new method involving the detection of ROS after a standardised singlet oxygen production at Day 1 (D1), 5 and 10 after SAH. Influence of RROS had been considered as a predictive tool of death at day 15 together with WFNS and Glasgow scoring, C-reactive protein, Protein S100B (P S100B) as well as aneurism location or the occurrence of a vasospasm and patients' history.

Results: Resistance to ROS at D1 was in $82 \%$ of cases greater than in healthy controls. In case of death a decrease of RROS was noted after D5 when it remained low but stable for others. It correlated with WFNS and Glasgow scoring. These changes correlated also with poor prognosis aneurisms locations and the occurrence of vasospasm. P S100B was an efficient initial marker of prognosis but RROS changes correlated better with survival along with survival time.

Conclusion: Resistance to ROS is strongly modified after an SAH and seems to be a metabolic marker of prognosis after an SAH providing additional data to clinical scores or pathologic markers of damages to brain matter. It could be used in the future to monitor real time changes induced by prescribed anti-oxidative medications.

\section{Introduction}

Intracranial aneurisms (ICA) are the main cause of subarachnoid hemorrhages $(\mathrm{SAH})$ and lead to a death toll reaching 40 to $60 \%$ of patients, 25 to $40 \%$ dying during the first week following their hospital admission. Among those who will survive 30 to $70 \%$ will suffer with various deficits. It remains difficult to establish an early prognosis and the need for biomarkers is therefore important to predict SAH outcome [1]. Oxidative stress, a major actor during ICA formation and rupture [2-3] results in the formation of reactive oxygen species (ROS) either primary including singlet oxygen $\left({ }^{1} \mathrm{O}_{2}\right)$ an excited form of ${ }^{1} \mathrm{O}_{2}$ or secondary including peroxides, and leads to the depletion of plasma physiological antioxidants causing additional tissues damage. ROS, generated during inflammatory reactions and/or energy production reactions, must deactivate leading to random deleterious events depending to some extent on each patient specific Resistance to Reactive Oxygen Species (RROS). In a preliminary study we noted that patient's RROS after strokes [4] strongly differed from what was observed during acute myocardial infarctions [5] and RROS also differed between ischemic strokes and SAH [6]. In parallel we noted that environmental factors influenced $\mathrm{SAH}$ onset in patients with
ICAs, cooperating with their general health status [7], thus suggesting that SAH onset could be linked to oxidative stress related [8-11] mechanisms [12]. Finally vasospasm which is often noted during the early hours after SAH is also potentially influenced by oxidative stress [13]. In the present paper we hypothesize that sensitivity to ROS may change with time after a SAH according to its severity, varying from one patient to another, thus influencing the treatment outcome. The aim of the present study was therefore to measure changes of RROS during the first 10 days following a SAH and evaluate whether RROS could represent a prognosis factor of death at 15 days, in addition to other markers. RROS was indirectly measured by analyzing the capability of a serum to deactivate a calibrated amount of exogenously produced ROS, in the present study after a photoreaction. If time for ROS deactivation is long, the total amount of ROS detected is high and RROS is low.

\section{Patients, materials and methods}

The study was conducted according to the protocol (NTS 200602) for the collection of healthy sera and was approved by the Nantes

*Correspondence to: Patrice Thierry, Laboratoire de Photobiologie, Anesthesiology and Intensive Care, PHU3, Laënnec Hospital, 44093 Nantes, France, Tel: (33) 2-40-16-56-75; Fax: (33) 2-40-16-59-35; E-mail: Thierry. Patrice@chu-nantes.fr

Key words: subarachnoid hemorrhage, intracranial cranial aneurysm, oxidative stress, singlet oxygen

Received: September 08, 2018; Accepted: September 25, 2018; Published: October 01,2018 
University Hospital ethics committee (HD-07/1519) in accordance with the Helsinki declaration (1964/2000).

Fifty Patients had been consecutively admitted in the intense care unit and data had been fully completed concerning the SAH circumstances as well as patient medical history. Diagnosis of SAH, origin and number of ICAs had been confirmed by NMR. All patients but one (Fisher score of 3 ) had a Fisher score of 4 . They were living at a maximum of $60 \mathrm{~km}$ around. Blood sampling had been performed on day 1 (D1), day 5 (D5) and day 10 (D10) after SAH onset. Evaluation of SAH was performed according to the WNFS and the Glasgow Coma Score (GCS). Patient's outcome had been evaluated at D15. Sequelae in survivors had not been considered for this preliminary study.

Clinical histories had been found extremely rich and we calculated for each patient a historical score representing the sum of all known diseases. Only patients unable to provide any marked souvenir of disease and without any clinical file were scored as 0 .

The SAH management followed the usual standards [14]. A brain CT scan confirmed diagnosis of aneurysmal SAH. Aneurysm was further confirmed during an arteriography during endovascular coiling procedure or surgical clipping in the first $24 \mathrm{~h}$ following patient's admission. Ventriculostomy was performed by the attending neurosurgeon in case of hydrocephalus. Patients with a GCS $<8$ were sedated with a continuous intravenous infusion of fentanyl $(2-5 \mu \mathrm{g}$ $\left.\mathrm{kg}^{-1} \mathrm{~h}^{-1}\right)$ or sufentanil $\left(0.2-0.5 \mu \mathrm{g} \mathrm{kg}^{-1} \mathrm{~h}^{-1}\right)$ and midazolam $(0.2-0.5 \mathrm{mg}$ $\mathrm{kg}^{-1} \mathrm{~h}^{-1}$ ) and were mechanically ventilated [15]. Cerebral perfusion pressure was maintained at $>60 \mathrm{mmHg}$ with norepinephrine [16]. Intracranial hypertension was defined as an intracranial pressure (ICP) over $25 \mathrm{mmHg}$ and treated with a bolus of mannitol $(0.5 \mathrm{~g} \mathrm{~kg}$ $\left.{ }^{1}\right)$ [15]. If ICP remained elevated after osmotherapy, barbiturates were administered (sodium thiopental) with an intravenous bolus of 2-3 $\mathrm{mg} \mathrm{kg}^{-1}$ followed by a continuous infusion of $2-3 \mathrm{mg} \mathrm{kg}^{-1} \mathrm{~h}^{-1}$. Sedation was stopped when patients were considered at low risk of intracranial hypertension. Nimodipine was intravenously administered on admission to the ICU (1-2 $\left.\mathrm{mg} \mathrm{h}^{-1}\right)$ and delivered via the enteral feeding tube as soon as enteral nutrition was delivered. Diagnosis of vasospasm was screened with transcranial Doppler twice a day (velocities above $120 \mathrm{~cm} / \mathrm{s}$ ) and diagnosis was upheld during arteriography by a trained neuroradiologist. Nimodipine was the first line of vasospasm treatment, angioplasty as a second line. Cardiovascular management was left to the attending physician's discretion depending on classical echo graphic parameters, pulse pressure variations and central pulse pressure [17].

Measurement of RROS had been described previously [18]. Briefly, a protoreaction is generated in a patients or a donor serum, producing a standardized amount of singlet oxygen $\left({ }^{1} \mathrm{O}_{2}\right)$. Then ${ }^{1} \mathrm{O}_{2}$ will deactivate with time into ROS that in turn will be detected by fluorimetry. Ten milliliters of venous blood were drawn on sterile clot act dry tubes (Venosafe VF-054SP, Terumo Europe, Leuven, Belgium) from donors as well as from patients. Hemolysis during blood sampling and hemoglobin aspiration during serum collection under sterile conditions were avoided as much as possible. Sera samples were frozen at $-20^{\circ} \mathrm{C}$ until processed. The period between blood sampling and freezing did not exceed 40 minutes. On the day of experiments sera were thawed to $0^{\circ} \mathrm{C}$, pooled and aliquoted in tubes of $0.5 \mathrm{~mL}$. Rose Bengal (RB, C.A.S [632-69-9], from Sigma-Aldrich, France) is added to sera $(5 \mu \mathrm{g} / \mathrm{mL}$ final concentration) in order to produce in the serum a standardized amount of photodynamically-induced ${ }^{1} \mathrm{O}_{2}\left({ }^{1} \mathrm{O}_{2}\right.$ quantum yield : $0.75,5$ $\mu \mathrm{g} / \mathrm{mL}$ final concentration) under light exposure (514 nm, $500 \mathrm{~mW}, 20$ $\mathrm{J} / \mathrm{cm}^{2}$ ) [19]. Singlet oxygen then deactivates by producing secondary
ROS. The speed of neutralization of ROS and/or peroxides is analyzed by means of the DCFH-DA/DCF system, activated DCFH being added to each sample assayed immediately after the end of light delivery and in a standardized manner. A control serum (prepared from a pool of sera collected from 75 healthy donors) and a non-photo-treated patient sample were processed in parallel to each patient sample. Finally, each measurement was the mean of a quadruplicate. The area under the curve (AUC) of the DCF fluorescence (excitation $488 \mathrm{~nm}$, emission $525 \mathrm{~nm}$ ) evolution following exposure to SOS over a period of 66 minutes was measured for each sample. In parallel Absorption spectra were obtained using a Techcomp 8500 absorption spectrophotometer (Techcomp, Honk Kong, China) after dilution in water for injections (5\% serum). Patient sera were discarded when absorbance was higher than 0.3 O.D. This occurred once in the present study. From preliminary data the mean ratios of (presumed) healthy donors was 1 and given the absence of other published data it was arbitrarily decided that the normal range was $\pm 10 \%$, i.e. a $20 \%$ range corresponding to $97 \%$ of values obtained in controls. However, we extended for patients this interval to $\pm 20 \%$ for safety reasons. A value higher than 1.2 indicated a greater AUC fluorescence than the reference pool used and thus a lower capacity for the given serum to neutralize ${ }^{1} \mathrm{O}_{2}$ and related SOS over time, whereas a value lower than 0.8 indicated a higher capacity. The more antioxidant the serum, the lower is the patient-over-control ratio.

C-Reactive protein values were obtained by immune-turbidimetry (mice antibodies on latex particles) by the mean of a COBAS $6000 \mathrm{c}$ Roche. Normal values were less than $5 \mathrm{mg} / \mathrm{L}$. Protein S100 B (P S100B) values were obtained by electro chemo luminescence (rutheniumlabelled mice antibodies plus biotinylated S 100 antibodies, streptavidin amplified) by the mean of a COBAS 6000 e Roche. Normal value (95 percentiles) was less than $0.105 \mu \mathrm{g} / \mathrm{L}$. Ratio neutrophils -to-lymphocytes ratio (NLR) [20-23] have been shown to be elevated during SAH and also acute myocardial infarction. Total white blood cells (WBC), absolute neutrophil count (ANC) and absolute lymphocyte count (ALC) were retrieved, and the NLR computed as the ratio of the ANC to ALC values.

Statistical analysis was thus based on the comparison of groups of individuals as a function of meteorological data. Chi-square test was used for the comparison of percentages, Mann-Whitney $U$ test for the comparison of meteorological data according to groups of patients, Spearman rank's test for correlations. Sensitivity expressed as a percentage or true positive rate was the number of True Positive patients $(\mathrm{TP})$ over $\mathrm{TP}+$ False negative patients $(\mathrm{FN})$ giving Sens $=\mathrm{TP} /$ $\mathrm{TP}+\mathrm{FN}$. Specificity was True Negative patients (TN) over TN+ False positive patients $(\mathrm{FP})$ giving $\mathrm{Spec}=\mathrm{TN} / \mathrm{TN}+\mathrm{FP}$.

\section{Results}

Among the 50 patients consecutively recruited for severe $\mathrm{SAH}$ related to an ICA disruption all data needed (location, timing, medical history i.e. and blood samples) for the present study were available in 49 (mean age 54.1, extremes 26-82). Patient or SAH characteristics of these 28 women $(57 \%)$ and 21 men $(p<0.05)$ are summarized in table 1. They did not differ from other published studies. The mean WFNS was 3.63 , mean GCS 8.45 , both slightly worse for women. Of these patients 9 were dead at 10 days and 20 (40.8\%), 11 women (39\%), at 15 days, death occurrence correlating with initial scoring.

Resistance to reactive oxygen species at D1 was found "better or greater" than the normal rate in $82 \%$ of cases, (the rate of ROS detected being lower than for controls), in the normal range for $18 \%$, none "worse" than controls. RROS at D1 inversely correlated with WFNS 
Table 1. Demographic data and risk factors of 49 patients with sub arachnoidal hemorrhage according to day 15 outcome

\begin{tabular}{|c|c|c|c|c|c|c|}
\hline & Total & Age & Female & Age & Male & Age \\
\hline All & 49 & 54.13 & $28(57.1)$ & 54.13 & $21(42.9)$ & 54.13 \\
\hline WFNS & 3.63 & - & 3.44 & - & 3.89 & - \\
\hline Glasgow & 8.45 & - & 8.69 & - & 8.14 & - \\
\hline Risk factors & - & & - & - & - & - \\
\hline BMI & 25.62 & - & 25.23 & - & 26.06 & - \\
\hline Smoking & NS 26, W 5, AS 17 & - & NS 9, W 4, AS 7 & - & NS $17, \mathrm{~W} 1$, AS 10 & - \\
\hline Stroke & Pers 3, Fam 5 & - & Pers 2, Fam 4 & - & Pers 1, Fam 1 & - \\
\hline Dead & $20(40.8)$ & 53.25 & $11(39)$ & 55.00 & $9(42)$ & 54.25 \\
\hline WFNS & 4.25 & - & 4.45 & - & 3.83 & - \\
\hline Glasgow & 8.67 & - & 9.10 & - & 7.33 & - \\
\hline Risk factors & - & - & - & - & - & - \\
\hline BMI & 24.90 & & 23.1 & & 26.10 & - \\
\hline Smoking & NS 9, W 0, AS 9 & - & NS 4, W 0, AS 6 & - & NS 5, W 0, AS 3 & - \\
\hline Stroke & Pers 1, Fam 4 & - & Fam 3 & - & Pers 1, Fam 1 & - \\
\hline Alive & $29(59.2)$ & 54.75 & $17(60)$ & 53.59 & $12(57)$ & 54.90 \\
\hline WFNS & 3.35 & - & 2.93 & - & 3.82 & - \\
\hline Glasgow & 8.41 & - & 9.33 & - & 7.36 & - \\
\hline Risk factors & - & - & - & & - & - \\
\hline BMI & 26.35 & - & 25.97 & - & 25.98 & - \\
\hline $\begin{array}{l}\text { Smoking } \\
\text { Stroke }\end{array}$ & $\begin{array}{l}\text { NS 17, W 5, AS } 8 \\
\text { Pers 2, Fam } 1\end{array}$ & - & $\begin{array}{l}\text { NS 5, W 4, AS } 1 \\
\text { Pers 1, Fam } 1\end{array}$ & - & $\begin{array}{l}\text { NS } 12, \text { W } 1, \text { AS } 7 \\
\text { Pers } 1\end{array}$ & - \\
\hline
\end{tabular}

Personal: Pers, Familial: Fam. Nonsmoking: NS, Withdrawn: W, Active smokers: AS.

$(p=0.001$, correlation coefficient -0.2$)$ when RROS at D10 positively correlated with WFNS $(p=0.02$, correlation coefficient 0.17$)$ and inversely with GCS ( $p=0.003$, correlation coefficient -0.2$)$. When mean D1 values of ROS detected were lower for patients with a poor outcome at D15, mean D10 values were significantly higher for patients with a bad WFNS or GCS, than for patients with a less severe scoring (Figure $1 \mathrm{~A}, 1 \mathrm{~B})$. This correlated well with the overall occurrence of death at 15 days (Figure 1C). For these patients' resistance to oxidative stress appears to be non-significantly greater at D1 $(p<0.06)$ but significantly lower at D10 than for alive patients, although remaining greater than for the healthy controls. Difference between D1-D10 values for patients with a poor prognosis is thus also significantly greater than for patients alive at 15 days.

Mean value of P S100B was higher at D1 in the group of patients with a poor prognosis when C-RP and RROS were found lower (Figure 2). However, when evolution of P S100B and C-RP was found nonlinear from D1 to D5 and D10, RROS decreased continuously in the group with a poor prognosis (Values of ROS detected increased, Figure 2B). This explains changes in sensitivity and specificity between D1 and D10 calculated in patients for RROS, C-RP, P S100B and neutrophil-to lymphocyte ratio (NLR) (Table 2). Sensitivity to predict SAH severity was found the highest with P S100B at D1 but decreased with time for all markers but RROS. Specificity to death was found comparable for $\mathrm{P}$ S100B and RROS if D1 to D10 values were all considered. Combination of P S100B and RROS provided a prognostic specificity and sensitivity to death of about $60 \%$. There was no correlation $(p<0.47)$ between evolution of RROS and the occurrence of respiratory complications (hypoxia defined as a $\mathrm{SaO}_{2}$ less than $90 \%$ for more than 1 hour) observed in $30 \%$ of cases, a rate comparable to others [24].

Aneurisms were located on ACOA in 13 (4 deaths at D15), MCA in 12 (6 deaths), ACA in 6 patients (4 deaths), posterior locations in 9 ( 2 deaths) and 9 various other anterior locations (4 deaths). A posterior location of an aneurism-related SAH induced the most decreased RROS at D5, then increasing when other locations i.e. ACA induced a constantly decreasing RROS (Figure 3A). A vasospasm [25] had been
Table 2. Sensitivity (Sens) and specificity (Spec) of biomarkers in 49 patients with a sub arachnoidal hemorrhage (SAH). Criteria were the prediction of SAH or of death after 15 days

\begin{tabular}{|c|c|c|c|}
\hline \% & Sens SAH & Sens to death & Spec to death \\
\hline CRP & 88 & 71 & 21 \\
\hline CRP evolution & NA & 66 & 50 \\
\hline NLR & 87 & 82 & 11 \\
\hline PS100B & 45 & 73 & 60 \\
\hline PS100B evolution & NA & 21 & 68 \\
\hline RROS & 82 & 94 & 36 \\
\hline RROS evolution & NA & 91 & 66 \\
\hline PS100B + RROS & NA & 60 & 56 \\
\hline
\end{tabular}

Sensitivity for SAH of C-Reactive protein: $\mathrm{CRP}(\mathrm{N}<5 \mathrm{mG} / \mathrm{L})$; Neutrophils-to-lymphocytes ratio: NLR $(2<\mathrm{N}<2.5)$; Protein S100 beta: PS100B $(\mathrm{N}<0.105 \mu \mathrm{G} / \mathrm{L})$, Resistance to reactive oxygen species: RROS, were calculated at D1.

« Evolution » results from calculated differences of values at D1 and D10

noted in 21 out of 49 patients (42\%) leading to death in 9 cases when 11 patients deceased without detected vasospasm (39\%). RROS in patients with vasospasm significantly increased at D5 as compared to D1 $(p<$ 0.01 ) but the slope of the curve was then similar to patients without vasospasm (figure 3B). Finally, proteins, glycaemia and leucocytes were lower at D1 in patients alive after 15 days, higher in others. RROS on the contrary was lower in patients with the worse prognosis (Figure 4).

It had not been possible to record any severe disease in $9(18 \%)$ out of 49 patients with a SAH (Figure 5) and 14 (28\%) have had more than $3 \mathrm{SAH}$-unrelated severe diseases. If death rate was comparable at around 30\% when less than 3 diseases had been recorded, it reached $50 \%$ when past diseases were more than 3 or on the contrary when no marked disease was recorded. Resistance to ROS detected at D1 after ${ }^{1} \mathrm{O}_{2}$ production was significantly relatively "worse", although greater than in the normal healthy controls when the number of diseases was null, "better" when diseases had been more frequent, reflecting a worse prognosis in patients having had more than 3 ROS-inducing severe diseases. Withdrawn smokers were more frequent in the "Alive" group and a previous history, familial or personal, of cerebral stroke was significantly $(p<0.05)$ more frequent $(16 \%)$ than prevalence at 50 in the French population (inferior to $4 \%$, classically $2.5 \%$ ). BMI was found 

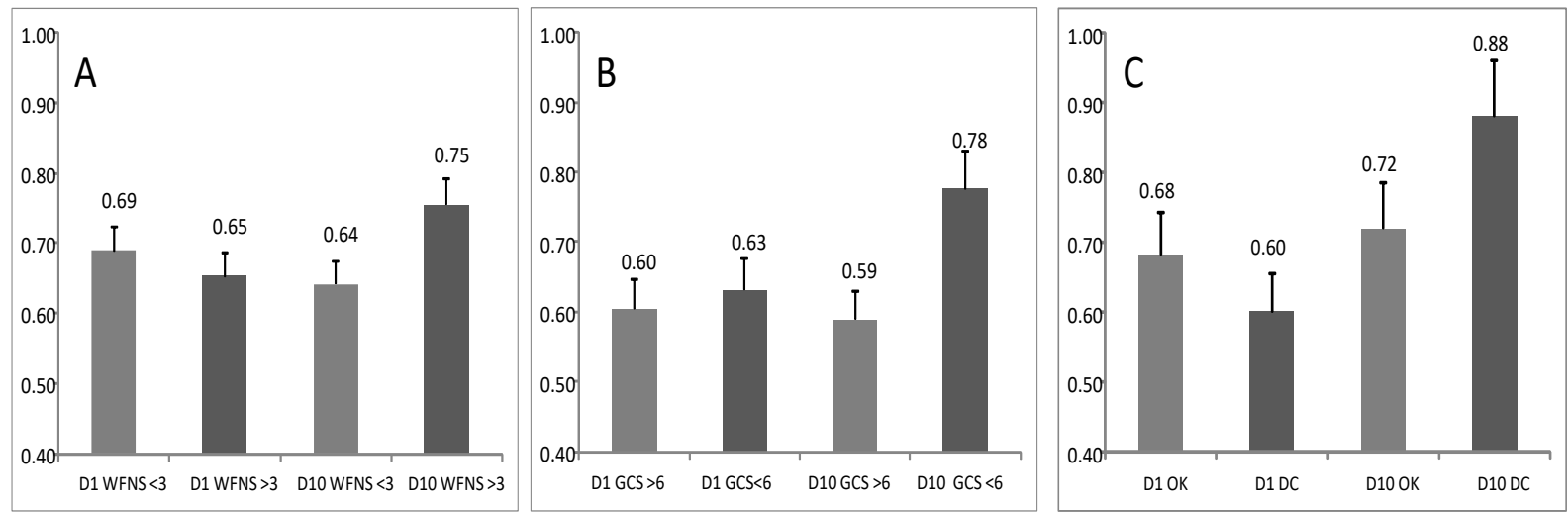

Figure 1. Reactive Oxygen Species detected after a calibrated photoreaction as compared to healthy controls (score 1) in 49 patients with subarachnoid hemorrhage at day 1 (D1) or day 10 (D10) according to the World Federation of Neurologic Surgeons score (WFNS, figure 1A), to the Glasgow coma score (GCS, figure 1B) or to an evolution to death (DC) or to survival at day $15(\mathrm{OK})$, figure $1 \mathrm{C}$.
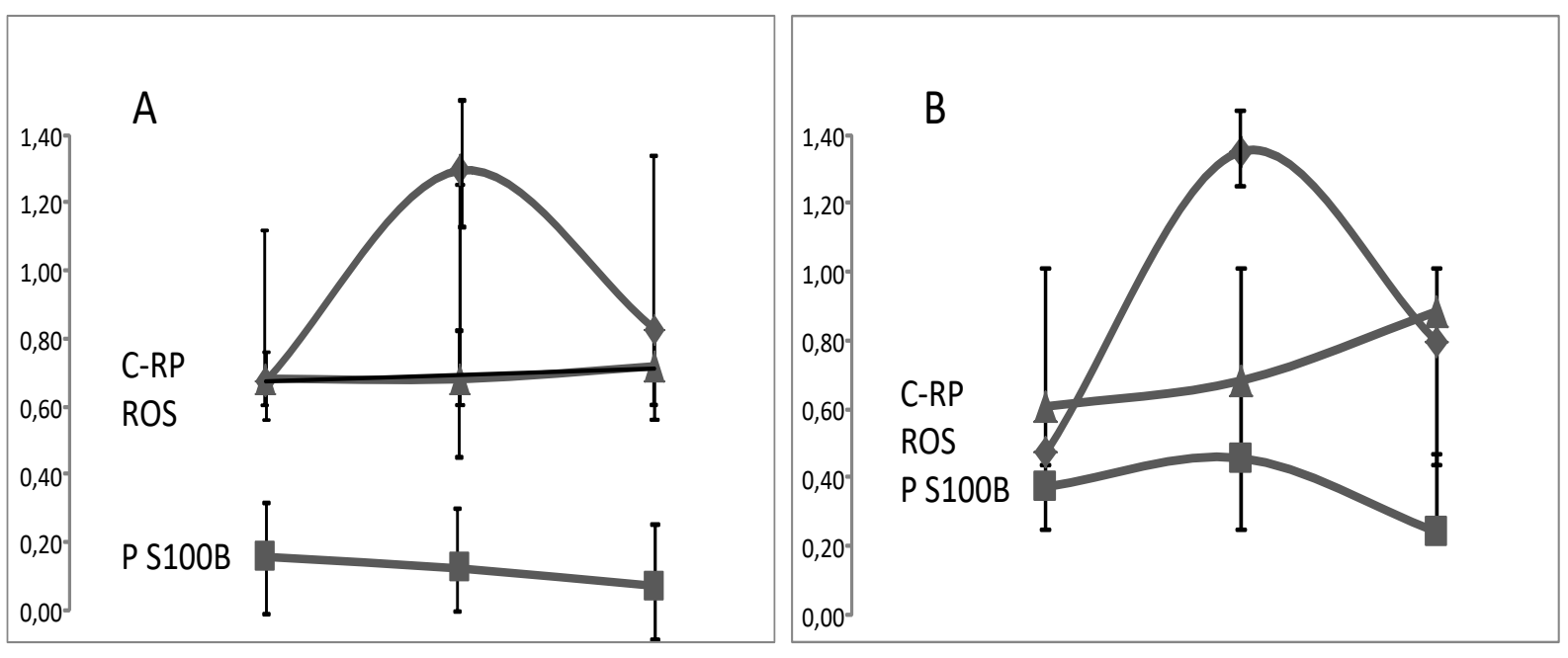

Figure 2. Evolution of C-Reactive Protein (CRP/100, $\bullet$ ), Protein S100 B (P S100B, $\boldsymbol{\square}$ ) and rate of Reactive Oxygen Species (ROS, $\mathbf{\Delta})$ detected after a calibrated photoreaction as compared to healthy controls measured at day 1, Day 5 and Day 10 after a Subarachnoid Haemorrhage in 49 patients alive at Day 15 (29 patients, figure 2A) or dead (20 patients, figure 2B).
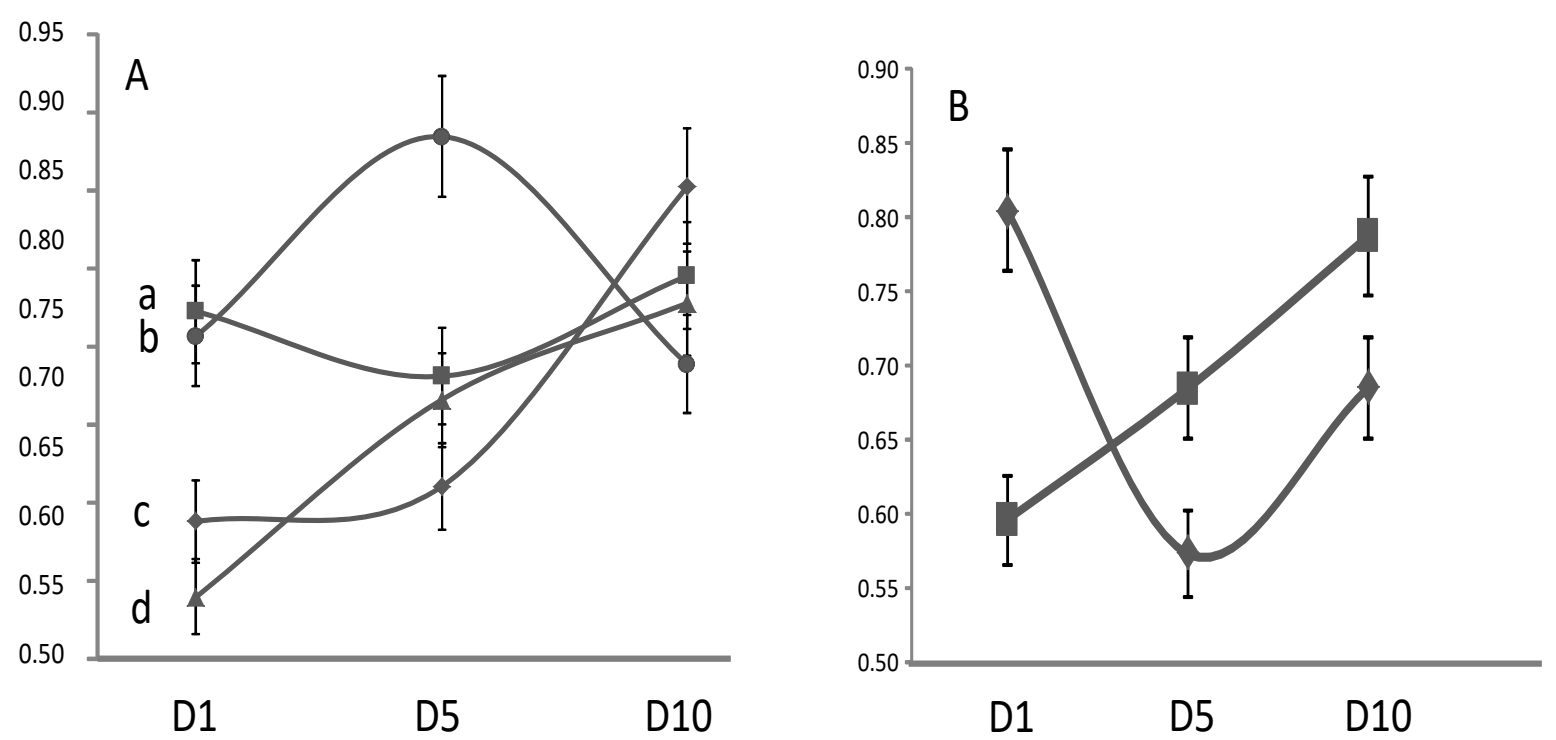

Figure 3. Influence of aneurysm location (A) or vasospasm (B) on the reactive oxygen species (ROS) detected after a calibrated photoreaction as compared to healthy controls (score 1) in 49 patients with subarachnoid hemorrhage at day 1 (D1), day 5 (D5) or day 10 (D10): a: anterior communicating artery (ACOA), b: Posterior locations, c: Middle cerebral artery (MCA), d: Anterior cerebral artery: (ACA). Evolution of ROS detection as compared to controls in patients without a vasospasm $(\mathrm{N}=28, \mathrm{n})$ or diagnosed with a vasospasm $(\mathrm{N}=21)$. 


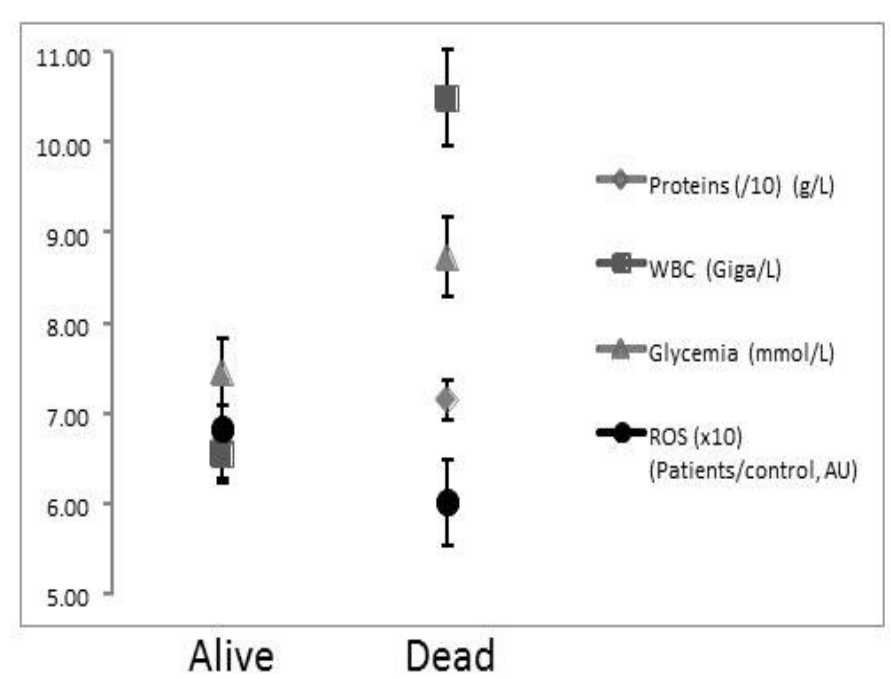

Figure 4. Glycaemia, white blood cells counts, total proteins and reactive oxygen species detected after a calibrated photoreaction as compared to healthy controls (score 1) measured at day 1 (D1) in the serum of 49 patients alive after a Subarachnoid Haemorrhage at day 15 (29 patients) or dead (20 patients).

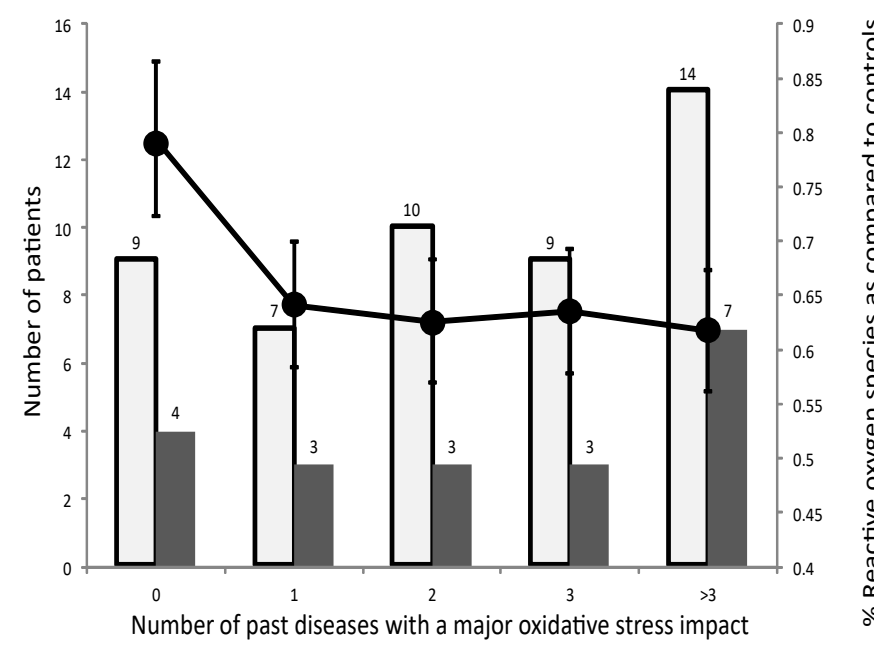

Figure 5. Influence of past diseases, likely to have induced a significant oxidative stress, diagnosed in 49 patients with subarachnoid hemorrhage on the number of deaths at day 15 and the Reactive Oxygen Species detected after a calibrated photoreaction as compared to healthy controls (score 1 ) measured at day $1(\bullet)$. Numbers are the number of patients in each group.

significantly higher than in the French population at 50 years old (BMI 18) but was similar in each subgroup.

\section{Discussion}

Subarachnoid hemorrhage from a ruptured aneurysm accounts for around $5 \%$ of all strokes. It has a high rate of mortality comprised between 40 and $60 \%$ according to studies and among survivors between 30 and $70 \%$ will suffer from heavy deficits $[26,27]$. Causes of aneurisms are not completely understood nor the causes of their disruption. When oxidative stress might play a role $[3,28]$ we demonstrated that RROS was decreased during ischemic strokes [4] and during a preliminary study that resistance to ROS was increased after SAH [6]. Characteristics of cohort recruited for the present study were found very similar to other papers SAH-related considering the sex ratio, risk factors, prognosis or treatment procedures $[29,30]$. As described earlier we noted that RROS at day 1 after SAH onset was significantly increased, only 4 patients ( 2 women) being within the arbitrarily estimated normal range of $1 \pm 15 \%$. However D1 or D10 calculation of RROS showed a perfect correlation with GCS [31], WFNS [32] scores or death rate. A relatively "greater" D1 RROS corresponded to the group of patients with a higher than 3 WFNS at D1 and a significantly lower RROS at D10 corresponded to a low GCS, a WFNS higher than 3 and a poor prognosis. C Reactive Protein [33] had been found significantly elevated at D1 in all patients, significantly higher at D5 but then decreasing rapidly to values close to initial ones whatever the patient outcome. A high D1 P S $100 \mathrm{~B}$ rate [34] was associated to a pejorative prognosis but was found to decrease at D10 whatever the prognosis, losing its prognostic value. This was not in agreement with [34] who found a better prognosis value at D15, but fit well to [35] who found a good prognostic value for P S 100B only at D1$\mathrm{D} 3$ or to [36,37]. RROS was non-significantly greater at D1 in patients with a poor prognosis $(p<0.06)$, remained unchanged in the group of patients alive at D15 but decreased (the rate of ROS detected increased) at D5 and D10 in the group of patient's dead at D15 $(p<0.01)$. Analysis of RROS was in agreement with the influence of aneurysm location on prognosis $[38,39]$ anterior locations being more frequent and often of worse prognosis at the exception of basilar ones. In our study, although values were a mix of good and poor outcomes, the only increased mean RROS at D10 was found for posterior locations $p<0.05$ as compared to COA, ACA and MCA.

During a vasospasm, that can be initiated itself by ROS, additional ROS are generated by hypoxia, inflammatory reactions [40] and enzymatic pathways $[2,41,42]$. According to literature data none of socalled classic biomarkers had been found able to detect the occurrence of vasospasm [36]. We cannot say that RROS measurement was able to do it but vasospasms induced significant changes between mean values noted at D1 and D5 $(p<0.01)$. In patients diagnosed with a vasospasm RROS strongly improved transiently at D5. As we noted earlier in the present paper that an initially relatively better RROS correlated to a more severe diagnosis, it suggests that a worsened prognosis was probable in these patients, their antioxidant defenses being even more solicited. More ischemia leading to more released ROS, one can hypothesize that additional circulating antioxidative defenses in the peripheral blood in reaction to a SAH are released, related to the vasospasm, throughout inflammatory pathways [43]. Therefore a greater consumption of antioxidative defenses would lead later to a pejorative prognosis merely in those having the lowest ones. For this reason, the rate of death is similar in the group having been diagnosed with a vasospasm and others.

Oxidative stress appears to be a major event during SAH as changes are noted in $82 \%$ of patients but contrarily to what had been noted in patients with an ischemic stroke [4] using the same above described method, SAH seems to induce a strong and early systemic reaction leading to an over RROS, then worsening in patients with a poor outcome. A reason could be the suddenness of the aneurysm disruption leading to a strong systemic reaction against ROS. As a result, when defenses are later eventually overtaken, there is a trend for RROS measurement to a return to the normal range, in fact corresponding to a pejorative event since ROS can no longer be neutralized till the end of the healing process. This hypothesis correlates well with RROS values measured according to aneurysm locations or vasospasm. This could also explain why a completely different evolution of RROS was observed after an ischemic stroke. In this latter case, patients being used to live with a poor RROS due to their health status, a vascular obliteration would lead to an additional consumption of antioxidants without any available compensation and therefore to a low RROS. RROS thus appears to be a non-specific value reflecting largely the patient's inflammatory status evolving inversely to WBC, glycaemia 
or proteins at least at early steps (D1). As noted in a previous study [7] there seems to be 2 categories of patients according to their D1 RROS and the number of severe ROS-generating diseases. In 9 patients an absence of diseases is correlated with a relatively low RROS at D1 when in others the RROS appears to be relatively "better" $(p<0.05)$. One could hypothesize that for patients already having been faced to oxidative stress, non-specific defenses are improved and rapidly mobilized. However, in a first attempt the rate of death does not seem to be influenced by the past diseases, indicating that early prognostic factors after an SAH are rather related to the aneurysm itself, which is in agreement with the prognostic value of WFNS or P S100 B value. $\mathrm{C}-\mathrm{RP}$ is a non-specific marker of the inflammatory reaction and is known to increase immediately after an SAH but due to treatments it rapidly normalizes. P S100B is a marker of neuronal damages and these damages are likely to extend only up to a certain limit. The biochemical expression of these thus should vanish with time, only reactivated by a vasospasm [34]. RROS on the contrary is a metabolic marker indicating that there is a local and a systemic reaction to various disorders SAHinduced. It is slower than others to be significant, but it correlates directly to the overall patient evolution, including when measured closely to death.

\section{Conclusion}

Response to ROS appears to be a major determinant of SAH outcome simply evidencing the metabolic capability of each patient to face to its own SAH. The number of false negatives does not increase with time suggesting that RROS could help monitoring patients and adapt therapies. These could eventually include antioxidant adjuvants in order to help the patient to overtake the critical days following SAH onset. The technology described in the present paper is easy to handle and could help to monitor RROS changes induced by treatments and adapt antioxidant doses.

\section{Acknowledgement}

We thank Dr. J. Ashton-Chess for English translation. We would thank by alphabetical order Byzance Charpentier, Tiphaine Chenon, Perrine Feaugas, medical students in the lab, for their help collecting data. Prof Thierry Patrice declare being the sole owner of the patent number PCT/FR2008/52042 describing the method used in the present paper therefore having a potential conflict of interest.

\section{References}

1. Hong CM, Tosun C, Kurland DB, Gerzanich V, Schreibman D, et al. (2014) Biomarkers as outcome predictors in subarachnoid hemorrhage--a systematic review. Biomarkers 19: 95-108. [Crossref]

2. Ayer RE, Zhang JH (2008) Oxidative stress in subarachnoid haemorrhage: significance in acute brain injury and vasospasm. Acta Neurochir Suppl 104: 33-41. [Crossref]

3. Starke RM, Chalouhi N, Ali MS, Jabbour PM, Tjoumakaris SI, et al. (2013) The role of oxidative stress in cerebral aneurysm formation and rupture. Curr Neurovasc Res 10: 247-255. [Crossref]

4. Ritzenthaler T, Lhommeau I, Douillard S, Cho TH, Brun J, et al. (2013) Dynamics of oxidative stress and urinary excretion of melatonin and its metabolites during acute ischemic stroke. Neurosci Lett 544: 1-4. [Crossref]

5. Guerin P, Bigot E, Patrice T (2013) Evidence for antioxidants consumption in the coronary blood of patients with an acute myocardial infarction. J Thromb Thrombolysis 35: 41-47.

6. Patrice T (2016) Photodynamic reactions as a prognostic tool for cardiovascular diseases. In: Hasan T, editor. Photodynamic therapy from bench to clinics. London: Royal Society of Chemistry 591-596.

7. Patrice T, Rozec B, Desal H, Blanloeil Y (2017) Oceanic Meteorological Conditions Influence Incidence of Aneurysmal Subarachnoid Hemorrhage. J Stroke Cerebrovasc Dis 26: 1573-1581. [Crossref]
8. Ruhnau J, Schulze J, Dressel A, Vogelgesang A (2017) Thrombosis, Neuroinflammation, and Poststroke Infection: The Multifaceted Role of Neutrophils in Stroke. J Immunol Res.

9. Davis SM, Pennypacker KR (2017) Targeting antioxidant enzyme expression as a therapeutic strategy for ischemic stroke. Neurochem Int 107: 23-32. [Crossref]

10. Duan X, Wen Z, Shen H, Shen M, Chen G (2016) Intracerebral Hemorrhage, Oxidative Stress, and Antioxidant Therapy. Oxid Med Cell Longev 2016: 1203285 [Crossref]

11. Aronowski J, Zhao X (2011) Molecular pathophysiology of cerebral hemorrhage: secondary brain injury. Stroke 42: 1781-1786. [Crossref]

12. Quillinan N, Herson PS, Traystman RJ (2016) Neuropathophysiology of Brain Injury Anesthesiol Clin 34: 453-464. [Crossref]

13. McMahon CJ, Hopkins S, Vail A, King AT, Smith D, et al. (2013) Inflammation as a predictor for delayed cerebral ischemia after aneurysmal subarachnoid haemorrhage. $J$ Neurointerv Surg 5: 512-517. [Crossref]

14. Diringer MN, Bleck TP, Claude Hemphill J, Menon D, Shutter L, et al. (2011) Critical care management of patients following aneurysmal subarachnoid hemorrhage: recommendations from the Neurocritical Care Society's Multidisciplinary Consensus Conference. Neurocrit Care 15: 211-240. [Crossref]

15. Rondeau N, Cinotti R, Rozec B, Roquilly A, Floch H, et al. (2012) Dobutamineinduced high cardiac index did not prevent vasospasm in subarachnoid hemorrhage patients: a randomized controlled pilot study. Neurocrit Care 17: 183-190. [Crossref]

16. Le Roux P, Menon DK, Citerio G, Vespa P, Bader MK, et al. (2014) Consensus summary statement of the International Multidisciplinary Consensus Conference on Multimodality Monitoring in Neurocritical Care: a statement for healthcare professionals from the Neurocritical Care Society and the European Society of Intensive Care Medicine. Intensive Care Med 40: 1189-1209. [Crossref]

17. Taccone FS, Citerio G (2014) Advanced monitoring of systemic hemodynamics in critically ill patients with acute brain injury. Neurocrit Care 2: S38-S63. [Crossref]

18. Olivier D, Douillard S, Lhommeau I, Bigot E, Patrice T (2009) Secondary oxidants in human serum exposed to singlet oxygen: the influence of hemolysis. Photochem Photobiol Sci 8: 1476-1486. [Crossref]

19. Redmond RW, Gamlin JN A (1999) compilation of singlet oxygen yields from biologically relevant molecules. Photochem Photobiol 70: 391-475. [Crossref]

20. Xue J, Huang W, Chen X, Li Q, Cai Z, (2017) et al. Neutrophil-to-Lymphocyte Ratio Is a Prognostic Marker in Acute Ischemic Stroke. J Stroke Cerebrovasc Dis 26: 650657. [Crossref]

21. Suh B, Shin DW, Kwon HM, Yun JM, Yang HK, et al. (2017) Elevated neutrophil to lymphocyte ratio and ischemic stroke risk in generally healthy adults. PLoS One 12: e0183706. [Crossref]

22. Lattanzi S, Cagnetti C, Provinciali L, Silvestrini M (2017) Neutrophil-to-lymphocyte ratio and neurological deterioration following acute cerebral hemorrhage. Oncotarget 8: 57489-57494. [Crossref]

23. Tokgoz S, Keskin S, Kayrak M, Seyithanoglu A, Ogmegul A (2014) Is neutrophil/ lymphocyte ratio predict to short-term mortality in acute cerebral infarct independently from infarct volume? J Stroke Cerebrovasc Dis 23: 2163-2168. [Crossref]

24. Ferdinand P, Roffe C (2016) Hypoxia after stroke: a review of experimental and clinical evidence. Exp Transl Stroke Med 8: 9. [Crossref]

25. Ciurea AV, Palade C, Voinescu D, Nica DA (2016) Subarachnoid hemorrhage and cerebral vasospasm - literature review. J Med Life 6: 120-125. [Crossref]

26. de Rooij NK, Linn FH, van der Plas JA, Algra A, Rinkel GJ (2007) Incidence of subarachnoid haemorrhage: a systematic review with emphasis on region, age, gender and time trends. J Neurol Neurosurg Psychiatry 78: 1365-1372. [Crossref]

27. Edjlali M, Rodriguez-Regent C, Hodel J, Aboukais R, Trystram D, et al. (2015) Subarachnoid hemorrhage in ten questions. Diagn Interv Imaging 96: 657-666. [Crossref]

28. Laaksamo E, Tulamo R, Liiman A, Baumann M, Friedlander RM, et al. (2013) Oxidative stress is associated with cell death, wall degradation, and increased risk of rupture of the intracranial aneurysm wall. Neurosurgery 72: 109-117. [Crossref]

29. Gruenbaum SE, Bilotta F (2014) Postoperative ICU management of patients after subarachnoid hemorrhage. Curr Opin Anaesthesiol 27: 489-493. [Crossref]

30. Nieuwkamp DJ, Rinkel GJ, Silva R, Greebe P, Schokking DA, et al. (2006) Subarachnoid haemorrhage in patients $>$ or $=75$ years: clinical course, treatment and outcome. J Neurol Neurosurg Psychiatry 77: 933-937. [Crossref] 
31. van Heuven AW, Dorhout Mees SM, Algra A, Rinkel GJ (2008) Validation of a prognostic subarachnoid hemorrhage grading scale derived directly from the Glasgow Coma Scale. Stroke 39: 1347-1348. [Crossref]

32. Teasdale GM, Drake CG, Hunt W, Kassell N, Sano K, Pertuiset B, et al. (1988) A universal subarachnoid hemorrhage scale: report of a committee of the World Federation of Neurosurgical Societies. J Neurol Neurosurg Psychiatry 51: 1457. [Crossref]

33. Juvela S, Kuhmonen J, Siironen J (2012) C-reactive protein as predictor for poor outcome after aneurysmal subarachnoid haemorrhage. Acta Neurochir (Wien) 154 397-404. [Crossref]

34. Sanchez-Pena P, Pereira AR, Sourour NA, Biondi A, Lejean L, et al. (2008) S100B as an additional prognostic marker in subarachnoid aneurysmal hemorrhage. Crit Care Med 36: 2267-2273. [Crossref]

35. Abboud T, Mende KC, Jung R, Czorlich P, Vettorazzi E, et al. (2017) Prognostic Value of Early S100 Calcium Binding Protein B and Neuron-Specific Enolase in Patients with Poor-Grade Aneurysmal Subarachnoid Hemorrhage: A Pilot Study. World Neurosurg 108: 669-675. [Crossref]

36. Moritz S, Warnat J, Bele S, Graf BM, Woertgen C (2010) The prognostic value of NSE and S100B from serum and cerebrospinal fluid in patients with spontaneous subarachnoid hemorrhage. J Neurosurg Anesthesiol 22: 21-31. [Crossref]
37. Oertel M, Schumacher U, McArthur DL, Kästner S, Boker DK (2006) S-100B and NSE: markers of initial impact of subarachnoid haemorrhage and their relation to vasospasm and outcome. J Clin Neurosci 13: 834-840. [Crossref]

38. Keedy A (2006) An overview of intracranial aneurysms. Mcgill J Med 9: 141-146. [Crossref]

39. Shapiro M, Becske T, Riina HA, Raz E, Zumofen D, et al. (2014) Toward an endovascular internal carotid artery classification system. AJNR Am J Neuroradiol 35: 230-236. [Crossref]

40. Miller BA, Turan N, Chau M, Pradilla G (2014) Inflammation, vasospasm, and brain injury after subarachnoid hemorrhage. Biomed Res Int 2014: 384342. [Crossref]

41. Friedman JK, Nitta CH, Henderson KM, Codianni SJ, Sanchez L, et al. (2014) Intermittent hypoxia-induced increases in reactive oxygen species activate NFATc3 increasing endothelin-1 vasoconstrictor reactivity. Vascul Pharmacol 60: 17-24. [Crossref]

42. Kim DE, Suh YS, Lee MS, Kim KY, Lee JH, et al. (2002) Vascular NAD(P)H oxidase triggers delayed cerebral vasospasm after subarachnoid hemorrhage in rats. Stroke 33: 2687-2691. [Crossref]

43. Lucke-Wold BP, Logsdon AF, Manoranjan B, Turner RC, McConnell E, et al. (2016) Aneurysmal Subarachnoid Hemorrhage and Neuroinflammation: A Comprehensive Review. Int J Mol Sci 17: 497. [Crossref]

Copyright: (C2018 Thierry P. This is an open-access article distributed under the terms of the Creative Commons Attribution License, which permits unrestricted use, distribution, and reproduction in any medium, provided the original author and source are credited. 